\title{
The binary neutron star merger rate via the modelled rate of short gamma-ray bursts
}

\author{
Debdutta Paul* \\ Tata Institute of Fundamental Research \\ E-mail: debdutta.paul@tifr.res.in
}

\begin{abstract}
Gamma-ray bursts (GRBs) are transients associated with the formation of compact objects. It had long been theorised that mergers of two neutron stars leading to the formation of a heavier neutron star or a black hole are the progenitors of the so-called 'short' GRBs. The merger is associated with the emission of gravitational waves (GWs) that are detectable on earth, and this association was proved empirically with the detection of a short GRB and other electromagnetic emission of the $\mathrm{GW}$ source 170817. It is important to make statistical predictions of the number of sGRBs detectable by a GRB monitor in the sky. Here I present predictions of the event rate of the AstroSat-CZTI via careful studies of the luminosity function of short GRBs. Using the maximum distance to which the GW networks are sensitive in the past, present and future runs, stringent lower limits are placed on the rate of binary neutron star mergers (BNSMs). It is shown that the number will go up significantly in the next observing runs of aLIGO/VIRGO. Comparison of the short GRB rate with the BNSM rate calculated independently from the single source statistics of GW170817 reveals the presence of a slight tension that can have significant implications on the physics of the merger ejecta; however the scenario that each BNSM indeed produces a short GRB, cannot be ruled out.
\end{abstract}

The New Era of Multi-Messenger Astrophysics - Asterics2019

25 - 29 March, 2019

Groningen, The Netherlands

${ }^{*}$ Speaker. 


\section{Introduction}

Although it is impossible to predict when a Gamma Ray Burst (GRB) is detected by a spacebased detector, it is nevertheless important to make statistical predictions of the number of GRBs detectable by a certain GRB monitor. Short GRBs (henceforth sGRBs) were theorised to originate from the environment of a pair of neutron stars at the verge of merging together, to form either a neutron star or a black hole, while emitting gravitational waves (GW). The detection of the GW source 170817 [1], a binary neutron star (henceforth NS) merger associated with a sGRB as well as other electromagnetic counterparts [2], sealed this association, putting the theory into empirical footing. In view of this, the rate of observable binary neutron star mergers (henceforth BNSMs) is linked to the rate of observable sGRBs. One can predict the BNSM rate by constraining the sGRB rate. This can be achieved by modelling the luminosity function (henceforth LF) of sGRBs, which will let us calculate the true sGRB rate of the universe. The direct modelling of the LF suffers from the limitation that there are too few GRBs with observed redshifts and hence estimated luminosities. In this work, I have applied the method followed by [3] to model the luminosity distribution of the full sample of sGRBs detected by BATSE, Fermi, and Swift till October, 2017. This is made possible by using a simplification proposed by [4] for long GRBs. I have then used the fitted models to calculate the true event rate of the sGRBs, and assuming that they are produced from BNSMs, deduced the rate of electromagnetic counterparts of gravitational wave events to which the GW detectors are sensitive in their different observing phases. All the catalogue data, scripts used and important databases generated are publicly available at https://github.com/DebduttaPaul/ luminosity_function_of_sGRBs.

\section{The short Gamma Ray Burst population}

\subsection{The luminosity function}

The validity of the Yonetoku correlation is first tested by combining all data from existing literature. The sGRBs, defined as $T_{90}<2.0$ seconds, have a smaller sub-sample of 15 with measured redshifts. Although the number of sources is small and there are at least three outliers, the correlation is found to be significant: a linear correlation coefficient of 0.98 is retrieved, and the hypothesis that it is generated from a random distribution is discarded (a probability of $8.9 \times 10^{-11}$ ). The best fit to the linear correlation is given by $L_{p}=(2.04 \pm 0.22) \times 10^{52} \mathrm{erg} \mathrm{s}^{-1}\left[\frac{E_{p, 0}}{\mathrm{MeV}}\right]^{1.17 \pm 0.18}$.

To model the LF of sGRBs, their luminosities are required for a large sample. The Yonetoku method of estimating luminosities via the pseudo-redshifts from the Yonetoku correlation is extended to include all sGRBs available in the catalogues of CGRO-BATSE, Swift-BAT, and Fermi-GBM. A total of 757 sGRBs are available up to GRB171025913. For all GRBs for which spectral parameter measurements are not available, the spectral energy peak, $E_{p}$, is randomly sampled from that of the observed distribution of Fermi GRBs with spectral measurements, following [4]. The justification behind this is: Fermi-GBM being a wide-band GRB detector, samples the $E_{p}$ space without selection bias, except at extremely high redshifts where the formation rate of GRBs is itself very small. Hence, the spectral parameter distribution of Fermi-GRBs is representative of the true GRB population. By randomly selecting $E_{p}$ from the observed distribution of Fermi 
Table 1: Comparison of the derived local GRB formation rate uncorrected for the beaming factor, $\dot{R}(0)$, with previous works.

\begin{tabular}{|c|c|}
\hline Reference & $\begin{array}{c}\dot{R}(0) \\
{\left[\mathrm{yr}^{-1} \mathrm{Gpc}^{-3}\right]}\end{array}$ \\
\hline \hline Ghirlanda et al. (2016), model [a] & $0.13-0.24$ \\
\hline Guetta \& Piran (2005) & $0.1-0.8$ \\
Yonetoku et al. (2014) & $0.24-0.94$ \\
Ghirlanda et al. (2016), model [c] & $0.65-1.10$ \\
present work & $0.61-3.89$ \\
\hline Coward et al. (2012) & $5-13$ \\
Guetta \& Piran (2006) & $8-30$ \\
\hline
\end{tabular}

bursts, the true distribution of $E_{p}$ of bursts is being sampled, and there is no need to additionally model this distribution. In doing so, no claim about the accuracy of the individual values of $E_{p}$ is claimed, and hence neither the individual values of pseudo-redshifts. This approach thus assigns pseudo-redshifts to bursts only in the statistical sense. This limitation is however not binding, since luminosities of the bursts estimated from pseudo-redshifts are used only as a collective sample in modelling the LF.

The number of sGRBs with known redshift and spectral parameters is only 15 . Hence, to test the hypothesis that the estimated pseudo-redshifts are indeed representative of the whole sample, I compare the cumulative distribution of the pseudo-redshifts thus derived for each of the instruments to that of the measured redshifts of a total of 30 sGRBs, with or without spectral parameters. The 2-sample KS test rules out the hypothesis that any of the pseudo-redshift distributions are drawn from the known redshift population when the full range of redshifts is considered. However, the number of GRBs with observed redshifts is still quite small to draw this negative conclusion, and the discrepancy can understood to be due to instrumental selection effects that severely limit the detection of GRBs with high redshifts, primarily via the identification of the host galaxy. [3] pointed out that the pseudo-redshift distributions matches well with the measured ones from their sample, when both are limited to a redshift of 1.0. Here it is found that as many as 25 of the 30 GRBs are located within this redshift. Given that the progenitor mass available for the production of sGRBs does not reduce drastically at $z>1.0$ from population synthesis studies, this is indicative of the fact that selection effects indeed play an important role in the measurement of redshifts of GRBs. When limited to this range, the pseudo-redshift distributions of all the instruments have probabilities $>0.66$ of being drawn from the same population as the known redshift distribution, hence the pseudo-redshifts can be safely used to calculate the luminosities for all the bursts with unknown redshifts. Studies that model the luminosity function considering only the short bursts with measured redshifts are not representative of the true population.

If the sGRB progenitors are produced by coalescences of NS binaries, then the effective mass available for coalescence per unit time per unit volume, is obtained by convolving the cosmic star formation rate with a powerlaw delay distributio, whose index is kept free. The simple powerlaw 
(SPL) model of the LF, $\Phi_{z}(L) \propto L^{-v}$, is ruled out for all three instruments within $v \in[0.01,10.0]$ with a high degree of confidence, contradicting [3], who found the LF to be well-described by a simple powerlaw of index 1, while supporting and extending the conclusion of [5], who ruled out this model with $v>2.0$. Next, the observed distributions are fit to the exponential cutoff powerlaw (ECPL) model with a break at $L_{\mathrm{b}}$, and the broken powerlaw (BPL) model with a break $L_{\mathrm{b}}$ separating two powerlaws with indices $v_{1}$ and $v_{2}$. It is seen that the low luminosity index $\left(v_{1}\right)$ of the BPL model is weakly constrained from below, although the other parameters are well constrained. The BPL model fits are consistent with the $68 \%$ confidence intervals quoted for this model by [5], for all three scenarios considered by them. From the current dataset, it is impossible to distinguish between the ECPL and BPL bestfit models, as the relative errors on the luminosity are large due to large propagated errors on the estimated luminosities (40\% on an average). From the normalisations of the parameters that fit the data, one retrieves the local sGRRB formation rate, $\dot{R}(0) \sim 0.61$ $3.89 \mathrm{yr}^{-1} \mathrm{Gpc}^{-3}$. A comparison with the recent literature is summarized in Table 1.

\subsection{Prediction for AstroSat-CZTI}

Combining the model parameters, predictions are made for the rate of sGRBs detectable by the AstroSat hard X-ray detector CZTI who predicted a sizeable under-detection for long GRBs. The combined model rate comes out in the range of $14-42 \mathrm{yr}^{-1}$ at $68 \%$ confidence. An updated list of GRBs available from the collaboration suggests that the detected number is $\sim 18 \mathrm{yr}^{-1}$, but these numbers are subjective, and also subject to change with time as the current method used is visual inspection of the data around the times that other satellites like Swift or Fermi have reported them. The searches are currently limited because of the lack of an automated GRB detection method, which depends on quantification of noise at the sub-second time-scales. An extensive work on this is under preparation, to be soon reported elsewhere.

\section{The binary coalescence rate}

The observed event rate of sGRBs can be corrected for the beaming factor $f_{\mathrm{B}}=1-\cos \left(\theta_{j}\right)$, where $\theta_{j}$ is the half-opening angle of the jet, to derive their true sky rate: $R_{0}=\frac{\dot{R}(0)}{f_{\mathrm{B}}}$. The conservative range of $3-26^{\circ}[6,7]$ is used to derive $R_{0}$. The $68 \%$ confidence ranges are given by $6.03-2838 \mathrm{yr}^{-1} \mathrm{Gpc}^{-3}$. Although the upper limit of $R_{0}$ is sensitive to the lower limit of $\theta_{j}$ and hence debatable, the lower limit of $R_{0}$ depends on the upper limit of $\theta_{j}$ and is hence fairly robust. Thus, a sharp lower limit of $R_{0} \sim 6 \mathrm{yr}^{-1} \mathrm{Gpc}^{-3}$ is placed with $68 \%$ confidence. Assuming that each BNSM produces a sGRB, this is also the minimum rate of BNSMs; if not, the merger rate is higher.

[8] gives the limiting distance $\left(D_{L}\right)$ versus inclination $(i)$ scatter plot with a detection criterion set to SNR > 8.0, for a combination of detectors: (a) the LH network comprising the Livingston and Hanford detectors, (b) the LHV network with the addition of the Virgo detector, and (c) the LHVKI network, including the KAGRA detector under construction in Japan, and the approved LIGO-India detector ${ }^{1}$ which is expected to come up in the next decade. I have used these, and integrated the $68 \%$ lower limit of $\dot{R}(z)$ upto the limiting redshift corresponding to $D_{L}$, to obtain the

\footnotetext{
${ }^{1}$ https://dcc.ligo.org/LIGO-M1100296/public
} 


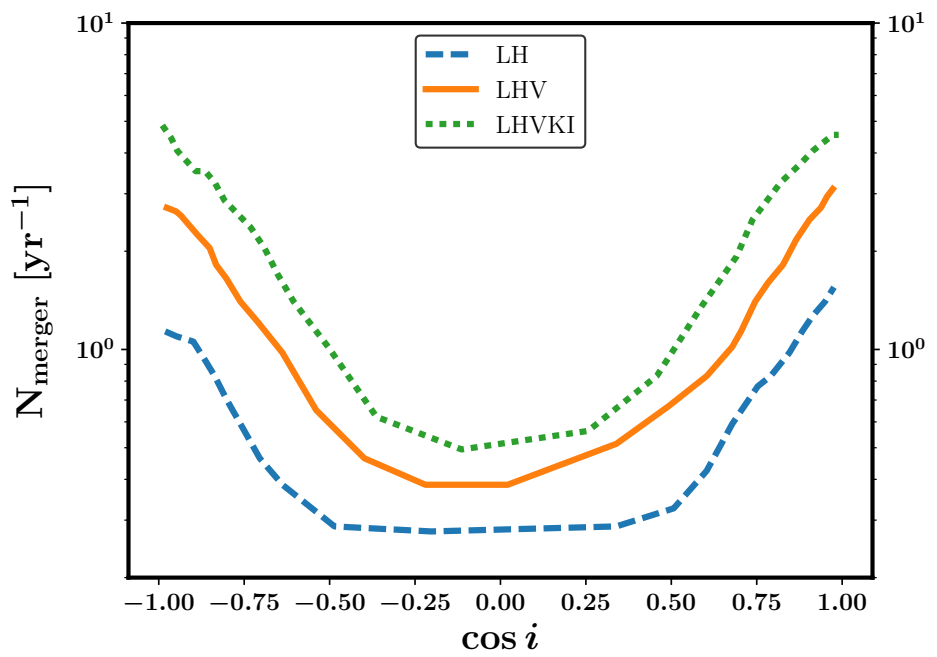

Figure 1: The lower limit of the binary neutron star merger rate (BNSM) as a function of the inclination of the normal to the merger plane with respect to the line of sight of the observer, $i$, for the LH (LivingstonHanford) configuration in (blue) dashed line, the LHV (Livingston-Hanford-Virgo) configuration in (orange) solid line, and the future LHVKI (Livingston-Hanford-Virgo-KAGRA-India) configuration in (green) dotted line. The data for the limiting distance of the GW networks used for this purpose have been taken from [8].

total rate as a function of $i$, shown in Fig. 1 . Giving equal weights to all $i$, the integrated rates are $0.95 \mathrm{yr}^{-1}$ for the LH network, $1.87 \mathrm{yr}^{-1}$ for the LHV network and $3.11 \mathrm{yr}^{-1}$ for the LHVKI network.

In the first two runs of the advanced LIGO, there has been only one confirmed detection of a neutron star binary merger, GW170817 [1]. The derived minimum integrated rate of $0.95 \mathrm{yr}^{-1}$ for the detection of BNSMs by the LH network is hence consistent with the detection of the single neutron star inspiral GW170817 that was extensively followed up across the electromagnetic spectrum (EM170817; [2]). In the ongoing O3 run, the number is expected to increase significantly, at the least to a few.

On the basis of the gravitational wave (GW) data alone from GW170817, [1] placed the rate of BNSMs at $320-4740 \mathrm{yr}^{-1} \mathrm{Gpc}^{-3}$ at $90 \%$ confidence. This rate is consistent but significantly higher than the sGRB rate derived in this work, $R_{0} \sim 6-2838 \mathrm{yr}^{-1} \mathrm{Gpc}^{-3}$. This implies that the fraction of GRBs produced from the BNSMs may be smaller than unity. This has important implications in the physics of the mergers: a fraction of the mergers may not be able to produce the classic onaxis jet that are hypothesized to cause sGRBs associated with the gravitational waves. The on-axis jet scenario has indeed been ruled out for GW/EM170817 by [9], who proposed a cocoon model to explain the multi-wavelength electromagnetic observations. Given the large uncertainties from both the GW and the sGRB rates, the relative ratio cannot be constrained well. As the ongoing $\mathrm{O} 3$ run is expected to significantly improve the detection of the BNSMs, it is envisaged that similar extensive follow-up campaigns of the electromagnetic counterparts of these mergers will shed more light on the physical processes surrounding the merger and the evolution of the associated ejecta. 


\section{References}

[1] B. P. Abbott, R. Abbott, T. D. Abbott, F. Acernese, K. Ackley, C. Adams et al., GW170817: Observation of Gravitational Waves from a Binary Neutron Star Inspiral, Physical Review Letters 119 (2017) 161101 [1710.05832].

[2] B. P. Abbott, R. Abbott, T. D. Abbott, F. Acernese, K. Ackley, C. Adams et al., Multi-messenger Observations of a Binary Neutron Star Merger, ApJ 848 (2017) L12 [1710 . 05833].

[3] D. Yonetoku, T. Nakamura, T. Sawano, K. Takahashi and A. Toyanago, Short Gamma-Ray Burst Formation Rate from BATSE Data Using $E_{p}-L_{p}$ Correlation and the Minimum Gravitational-wave Event Rate of a Coalescing Compact Binary, ApJ 789 (2014) 65 [1402 . 5463].

[4] D. Paul, Modelling the luminosity function of long gamma-ray bursts using Swift and Fermi, MNRAS 473 (2018) 3385 [1709.09145].

[5] G. Ghirlanda, O. S. Salafia, A. Pescalli, G. Ghisellini, R. Salvaterra, E. Chassande-Mottin et al., Short gamma-ray bursts at the dawn of the gravitational wave era, A\&A 594 (2016) A84 [1607.07875].

[6] W. Fong, E. Berger, R. Margutti and B. A. Zauderer, A Decade of Short-duration Gamma-Ray Burst Broadband Afterglows: Energetics, Circumburst Densities, and Jet Opening Angles, ApJ 815 (2015) 102 [1509.02922].

[7] R. Margutti, E. Berger, W. Fong, B. A. Zauderer, S. B. Cenko, J. Greiner et al., The Afterglow and Environment of the Short GRB 111117A, ApJ 756 (2012) 63 [1205.7075].

[8] M. Saleem, A. Pai, K. Misra, L. Resmi and K. G. Arun, Rates of short-GRB afterglows in association with binary neutron star mergers, MNRAS 475 (2018) 699 [1710.06111].

[9] M. M. Kasliwal, E. Nakar, L. P. Singer, D. L. Kaplan, D. O. Cook, A. Van Sistine et al., Illuminating gravitational waves: A concordant picture of photons from a neutron star merger, Science 358 (2017) 1559 [1710.05436]. 